\title{
Synthesis of daunomycin-oligoarginine conjugates and their effect on human leukemia cells (HL-60)
}

\author{
Zoltán Bánóczi, ${ }^{\mathrm{a}, \mathrm{b}}$ Balázs Peregi, ${ }^{\mathrm{a}}$ Erika Orbán, ${ }^{\mathrm{a}}$ Rita Szabó,, ${ }^{\mathrm{a}, \mathrm{b}}$ and Ferenc Hudecz ${ }^{\mathrm{a}, \mathrm{b}^{*}}$ \\ ${ }^{a}$ Research Group of Peptide Chemistry, Eötvös L. University Hungarian Academy of Sciences, \\ P.O.Box 32, H-1518 Budapest 112 Hungary \\ ${ }^{b}$ Department of Organic Chemistry, Eötvös Loránd University, Budapest, Hungary \\ E-mail:fhudecz@,ludens.elte.hu
}

Dedicated to Professor Csaba Szántay on the occasion of his 80th birthday

\begin{abstract}
The synthesis of new daunomycin-conjugates, i.e. succinyl derivative of daunomycin (DauSuc) coupled to oligoarginine ( $\operatorname{Arg}_{n}, n=4,6$ or 8 ) is described. These compounds are stable to certain conditions used for in vitro cellular experiments. All conjugates depending on the number of Arg residues possess antiproliferative effect against sensitive and MDR1 resistant human leukemia (HL-60) cells. DauSucArg 8 and DauSucArg 6 were more efficient than free DauSuc. Uptake studies show that conjugates are entering both sensitive and MDR1 resistant HL-60 cells.
\end{abstract}

Keywords: Daunomycin, oligoarginine, cell-penetrating peptide, daunomycin conjugates, antitumor effect

\section{Introduction}

Anthracyclines (e.g. daunomycin (Figure 1.), doxorubicin, epirubicin, idarubicin and mitoxantrone) are widely used antineoplastic agents in the treatment of various types of cancer; like osteosarcomas and acute myeloid leukemia. ${ }^{1,2}$ 


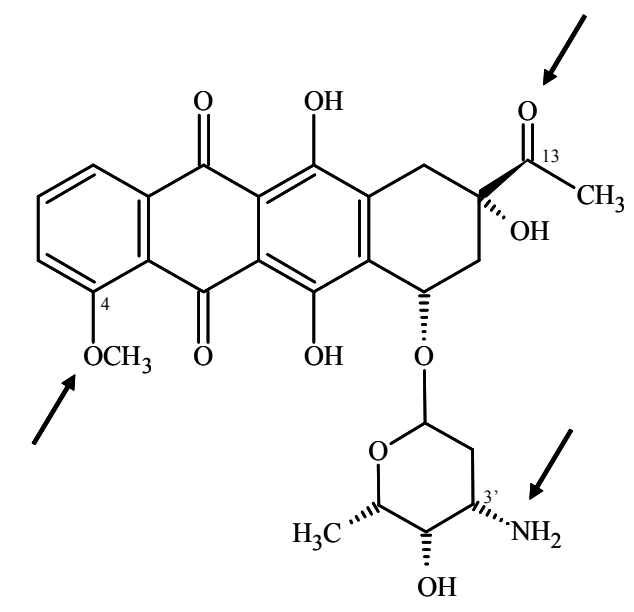

Figure 1. Structure of daunomycin and possible sites of conjugation.

Daunomycin (Dau) has side effects, like cardiotoxicity ${ }^{3}$ and its administration frequently results in resistance of tumor cells. ${ }^{4}$ In the development of multidrug resistance (MDR), different factors could play essential role(s). For example the increased drug efflux ${ }^{5}$ is often correlates with the over-expression of certain transporter proteins like P-glycoprotein (MRP1). ${ }^{6}$ To overcome MDR new strategies were developed. One of these is the co-administration of the drug with an inhibitor of transporter protein(s). ${ }^{6}$ Conjugation with oligopeptides (e.g. cell-penetrating ${\text { peptides })^{7} \text { or macromolecules }}^{8,9}$ can be considered as a novel, alternative approach.

Dau has three suitable functions for covalent conjugation (Figure 1). Replacement of the methyl ether group on C-4 is one of the possibilities. This strategy was used recently for coupling of Dau to triplex forming oligonucleotide(s). ${ }^{10}$ A second option for attachment is provided by the presence of a primary amino group of the sugar moiety of Dau (Figure 1$)^{11}$. Modification of 13-oxo group in the daunomycinone part (Figure 1) by hydrazine, $O$-alkyl hydroxylamine was also successfully applied in early experiments for amino acid and dipeptide coupling. ${ }^{11-13}$ Dau can be conjugated to carriers by direct or indirect strategy. In the direct strategy the amino group of Dau could be utilized for connection with amino acids and peptides. ${ }^{11-13}$ Inclusion of spacer/linker moiety is frequently applied. Dau was conjugated with Neuropeptide Y modified by Cys at position 15 using maleimide linker to target neuroblastoma cells. ${ }^{14}$ Maleimide linker was also coupled to Dau either on the 13-oxo group via an acidsensitive hydrazone bond or on the primary amino group via a stable amide bond. Dau also was attached to the $C$-terminal $\alpha$-helix peptide of human melanoma growth stimulating factor via a polyethylene glycol (3400) dialdehyde linker to target melanoma. ${ }^{15}$ Dau modified with either levulinic acid or 5-oxo-hexanoic acid was used to link to completely unprotected peptide fragments via oxime bond. ${ }^{16}$ Human calcitonin was coupled with Dau via an maleimide linker. In this construct either an acid-stable amide bond on the primary amino group or an acid-labile hydrazone bond on the 13-oxo group was formed. ${ }^{17}$ Conjugates were synthesized by 
incorporation of leucyl or aspartyl ${ }^{18}$ maleyl ${ }^{19}$ or cis-aconityl $1{ }^{19,20}$ succinyl or several diacidic ${ }^{21}$ spacer and different maleimide linkers. ${ }^{14,17,22}$

Cell-penetrating peptides could deliver covalently attached bioactive cargo into various cell types. ${ }^{23}$ Besides the oligopeptides of natural origin, synthetic peptides were also identified as efficient carriers. Oligoarginines represent a group of de novo designed cell-penetrating peptides. ${ }^{24,25}$ Studies of fluorescent oligoarginines suggested that the optimal chain length is six to eight Arg residues. ${ }^{24,26}$ It is interesting to note that only a limited number of studies was reported on the synthesis and biological activity of oligoarginine conjugated with potentially therapeutic compound (cyclosporin $\mathrm{A}^{27}$ and ferrocene derivatives ${ }^{28}$ ).

In this paper we describe the synthesis of a new group of daunomycin-oligoarginine $\left(\operatorname{Arg}_{\mathrm{n}}\right.$, $\mathrm{n}=4,6$ or 8) conjugates (Figure 2.) in which a succinyl derivative of Dau is coupled to the oligopeptide. Our data reported here, suggest that all conjugates possess antiproliferative effect against sensitive and resistant human leukemia (HL-60) cells in vitro. Uptake studies outlined show that conjugates are entering both sensitive and resistant HL-60 human leukemia cells.

\section{Results and Discussion}

Dau was coupled to oligoarginine with different length (Figure 2.) using a succinyl spacer between the two partners. The oligopeptide part of the conjugate contained 4, 6 or 8 Arg residues, while Dau was succinylated at the primary amino group of the daunosamine moiety. An amide bond was formed between the free carboxy function of the modified drug and the $\mathrm{N}$ terminal amino function of the oligoarginine. Conjugates were purified, characterized and their stability was studied under conditions used for certain biological experiments. In comparative studies we have analyzed the cytostatic effect of these compounds, their components in vitro as well as the cellular uptake of the constructs by both sensitive and resistant human leukemia (HL60) cells.<smiles></smiles>

Figure 2. Structure of conjugate with oligoarginine. 


\section{Synthesis and characterization of oligoarginine conjugates of daunomycin}

Since Dau is sensitive to acidic conditions, which are generally used for cleavage of peptides from the solid support, the conjugation of Dau derivative with oligoarginine peptide was achieved in solution. Thus oligoarginines were synthesized on solid supports by $\mathrm{Fmoc} / \mathrm{Bu}$ strategy. The peptides were cleaved from the resin by trifluoroacetic acid (TFA). Cleavage mixtures containing appropriate scavengers were purified by RP-HPLC (Table 1.). For the introduction of the carboxy function, the daunosamin part of Dau was modified by succinylation. In addition, this group can operate as a spacer providing a distance between the drug and the peptide moiety. Dau was reacted with succinic anhydride in dimethylformamide (DMF) in the presence of $N, N$-diisopropylethylamine (DIEA) as base. The succinylated daunomycin (DauSuc) was purified by RP-HPLC and characterized by MS. In the conjugation reaction, the $N$-terminal amino group of oligoarginine amides was coupled with DauSuc possessing a free carboxy function. Under conditions used in this study, oligoarginine amides with free side chains were used without any difficulties. The amide bond between the two partners was formed in DMF using tris(dimethylamino)phosphonium-hexafluorophosphate/1-hydroxybenzotriazole $(\mathrm{BOP} / \mathrm{HOBt})^{29}$ coupling reagents. The conjugates were purified by RP-HPLC and were characterized by analytical RP-HPLC and MS (Table 1.).

Table 1. Characteristics of oligoarginines and Dau-conjugates

\begin{tabular}{lccc}
\hline \multicolumn{1}{c}{ Compound } & $\mathrm{R}_{\mathrm{t}}(\mathrm{min})^{\mathrm{a}}$ & \multicolumn{2}{c}{$[\mathrm{M}+\mathrm{H}]^{+, \mathrm{b}}$} \\
\cline { 3 - 4 } & & $\mathrm{M}_{\text {cal. }}$ & $\mathrm{M}_{\text {meas. }}$ \\
\hline $\mathrm{Arg}_{4}$ & 14.7 & 642.7 & 642.5 \\
$\mathrm{Arg}_{6}$ & 15.3 & 955.1 & 954.9 \\
$\mathrm{Arg}_{8}$ & 15.7 & 1267.4 & 1267.5 \\
DauSucArg$_{4}$ & 27.3 & 1251.8 & 1251.8 \\
DauSucArg & 26.2 & 1564.2 & 1564.6 \\
DauSucArg $_{8}$ & 25.8 & 1876.9 & 1876.8 \\
\hline
\end{tabular}

${ }^{\mathrm{a} C}$ Column: Phenomenex Jupiter C18 (250x4.6 mm, $5 \mu \mathrm{m}, 300 \AA$ ); eluent A: 0.1\% TFA/ $\mathrm{H}_{2} \mathrm{O}, \mathrm{B}$ : $0.1 \% \mathrm{TFA} /$ Acetonitrile- $\mathrm{H}_{2} \mathrm{O}(80: 20 \mathrm{v} / \mathrm{v})$; gradient: 0 min $0 \% \mathrm{~B} ; 5 \min 0 \% \mathrm{~B} ; 50 \mathrm{~min} 90 \% \mathrm{~B}$; flow rate: $1 \mathrm{~mL} / \mathrm{min} .{ }^{\mathrm{b}} \mathrm{ESI}-\mathrm{MS}$

\section{Stability of oligoarginine conjugates of daunomycin}

The stability of conjugates was studied under conditions used for certain biological experiments. The samples were incubated for $180 \mathrm{~min}$ at room temperature and then analyzed by chromatography. Data obtained suggest that no change occurred in the chromatogram after $3 \mathrm{~h}$ (data not shown). These results indicate that conjugates could be considered as being essentially stable under conditions applied. 


\section{In vitro cytostatic effect of oligoarginine conjugates of daunomycin}

The antitumor effect of conjugates as well as free Dau and DauSuc was studied on sensitive and resistant HL-60 cells in vitro. The cells were incubated with a solution of compounds $(\mathrm{c}=2.6 \mathrm{x}$ $10^{-4}-10^{2} \mu \mathrm{M}$ ) for $3 \mathrm{hrs}$. After $72 \mathrm{hrs}$ the percent of living cells was measured by MTT assay. The level of cytostasis (\%) caused by the treatment, was measured as a function of concentration. Based on these curves the $\mathrm{IC}_{50}$ values were determined and are outlined in Figure 3. As expected, on sensitive HL-60 cells Dau has a high cytostatic effect as indicated by the low $\mathrm{IC}_{50}$ value $\left(\mathrm{IC}_{50}\right.$ $=0.05 \mu \mathrm{M})$. In case of DauSuc the effect is less marked $\left(\mathrm{IC}_{50}=8.31 \mu \mathrm{M}\right)$. This observation could be explained by the succinylation. As a consequence of this structural change, the positively charged amino group is now replaced by negatively charged carboxylic group under physiological conditions. Our data clearly show that this alteration results in 100 times lower antitumor effect under in vitro conditions.

The Dau-conjugates of oligoarginines exhibited cytostatic effect on sensitive HL-60 cells (Figure 3). However, this was influenced by the number of Arg residues present. The tetraarginine conjugate showed the highest $\mathrm{IC}_{50}$ value $\left(\mathrm{IC}_{50}=24.5 \mu \mathrm{M}\right)$, whereas the increasing chain length resulted in higher cytostatic effect; $\mathrm{IC}_{50}$ values of the hexa- and octaarginine conjugates were $8.4 \mu \mathrm{M}$ and $5.2 \mu \mathrm{M}$, respectively. It should be noted that the efficacy of DauSucArg $_{8}$ conjugate on sensitive HL-60 cells was slightly more expressed than that of the succinylated Dau $\left(\mathrm{IC}_{50}=5.2 \mu \mathrm{M}\right.$ vs $\left.\mathrm{IC}_{50}=8.31 \mu \mathrm{M}\right)$. These data suggest that the in vitro cytostatic effect of conjugates is dependent on the number of Arg residues present. Data show that the in vitro effect of the octaarginine conjugate is comparable with that of free DauSuc.

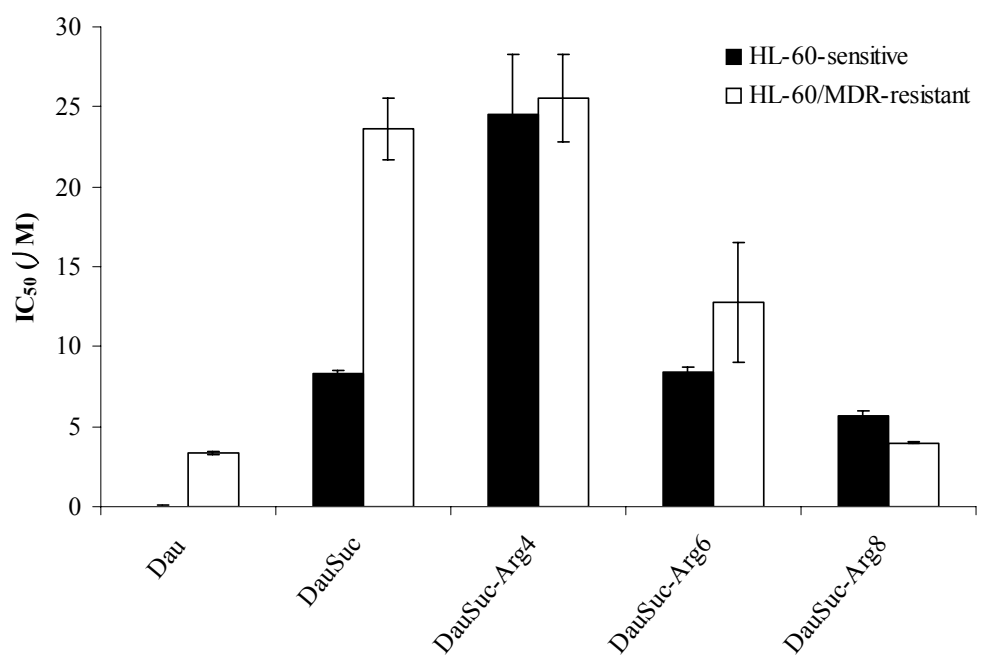

Figure 3. The comparison of the in vitro antitumor effect of compounds; Dau, DauSuc, DauSucArg $_{4}$, DauSucArg $_{6}$ and DauSucArg 8 on sensitive and resistant HL-60 human leukemia cells. Cells were treated with samples in the range of $c=2.6 \times 10^{-4}-10^{-2} \mu \mathrm{M}$. Based on cytostasis $\%$ measured by MTT-assay the $\mathrm{IC}_{50}$ values were determined. 
The Dau-conjugates were cytostatic also on resistant HL-60/MDR1 cells (Figure 3). In this case, the effect of free Dau was reduced by 3 -fold $\left(\mathrm{IC}_{50}=3.31 \mu \mathrm{M}\right)$ as compared of its activity on sensitive HL-60 cells. This confirms our previous findings ${ }^{29}$ that Dau is a very good substrate of MDR1 transport protein. Our data with DauSuc indicate that succinylation not only decrease the inhibitory effect of Dau, but this modification results in a derivative which is a poor substrate of MDR1 protein. DauSuc proved to be less efficient under in vitro conditions on HL-60/MDR1 cells than on sensitive leukemia cells $\left(\mathrm{IC}_{50}=23.7 \mu \mathrm{M}\right.$ for HL-60/MDR 1 cells vs $\mathrm{IC}_{50}=8.31 \mu \mathrm{M}$ for sensitive HL-60 cells).

The effect of Dau-conjugates were cytostatic on resistant HL-60/MDR1 cells too (Figure 3). This effect was also dependent on the number of Arg residues present in the peptide chain (Figure 3). DauSucArg 4 conjugate was the less cytostatic among conjugates studied $\left(\mathrm{IC}_{50}=25.6\right.$ $\mu \mathrm{M})$. Conjugates with six Arg residues exhibited lower $\mathrm{IC}_{50}$ value $\left(\mathrm{IC}_{50}=12.8 \mu \mathrm{M}\right)$, while the presence of octaarginine resulted in the highest cytostatic effect $\left(\mathrm{IC}_{50}=4.0 \mu \mathrm{M}\right)$.

In comparison with activity on sensitive cells, the conjugates with a different number of Arg residues almost fully preserved their cytostatic effect on resistant HL-60/MDR1 cells. The effect of tetra- and hexaarginine conjugates was slightly lower $\left(\mathrm{IC}_{50}=24.5\right.$ and $8.4 \mu \mathrm{M}$ for sensitive and $\mathrm{IC}_{50}=25.6$ and $12.8 \mu \mathrm{M}$ for resistant cells, respectively). Perhaps the most important finding of

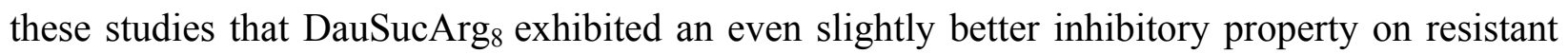
HL-60 cells $\left(\mathrm{IC}_{50}=5.2 \mu \mathrm{M}\right.$ for sensitive and $\mathrm{IC}_{50}=4.0 \mu \mathrm{M}$ for resistant HL-60/MDR1 cells, respectively).

The results summarized above with conjugates suggest that these compounds (a) are essentially no substrate of MDR1 transfer protein and/or (b) could use another internalization pathway than daunomycin and therefore the MDR1 protein can not pump them out from the cell. In both sensitive and resistant HL-60 cells the effect of conjugates was dependent on the number of Arg residues present. These findings are in harmony with our previous results using ferrocenecarboxylic acid as a drug in oligoarginine conjugates. ${ }^{28} \mathrm{We}$ found that a conjugate with eight Arg residues is the most effective one, but the differences between DauSucArg 8 and DauSucArg $_{6}$ are not so pronounced. The effect of both conjugates was similar with that of free DauSuc. It is important to emphasize that all conjugates studied were effective not only on sensitive, but also on MDR1 resistant cells.

\section{Cellular uptake properties of oligoarginine conjugates of daunomycin}

Fluorescence properties of Dau could be preserved even after conjugation at the daunosamine function. ${ }^{31}$ Therefore fluorescence intensity measurements could be used for studying the internalization of conjugates. Cell-uptake was examined also on sensitive and resistant HL$60 /$ MDR 1 cells using flow cytometry. First cells were incubated with the solution $(\mathrm{c}=30 \mu \mathrm{M})$ of tetra- or octaarginine conjugates for $90 \mathrm{~min}$, and fluorescence intensity of cells - proportional with the amount of internalized conjugates - was recorded. Experiments were performed with native and also with trypsin treated cells. Since conjugates comprise oligoarginine, these compounds are highly positively charged, and have marked tendency to bind to the cell 
membrane by electrostatic interactions. ${ }^{25}$ It was previously reported that trypsin digests the protein and peptide on the outer surface of membrane, so the measurement of fluorescence intensity of trypsin treated cells corresponds only to conjugates present inside the cells. ${ }^{32}$ In order to exclude artifacts we have first determined the amount of membrane bound conjugates by comparing the fluorescence intensity after incubation of cells with or without treatment of trypsin. Results obtained for both conjugates on sensitive HL-60 cells are shown in Figure 4.

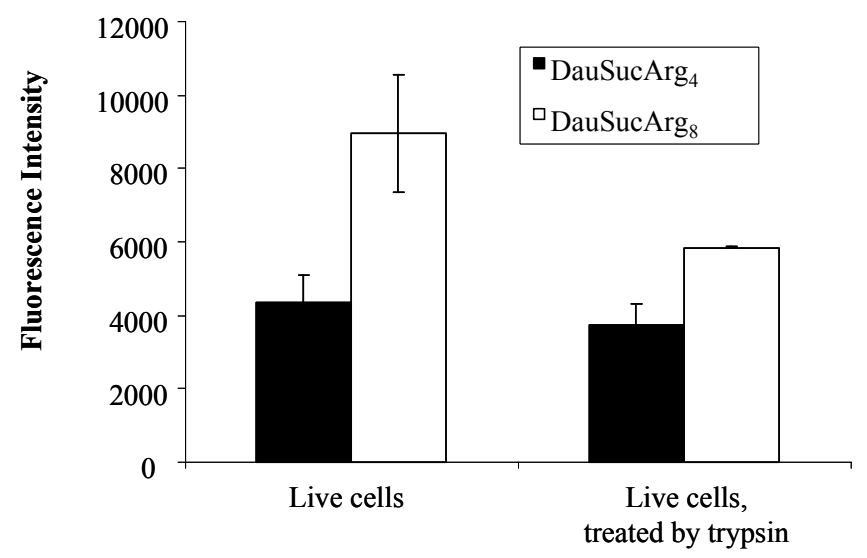

Figure 4. Mean fluorescence intensity of sensitive HL-60 cells incubated with conjugates before and after trypsin treatment.

Fluorescence intensity of cells incubated with DauSucArg ${ }_{4}$ conjugate was similar in both cases. However, the intensity of the fluorescence signal derived from cells incubated with DauSucArg $_{8}$ conjugate without trypsin treatment was higher than that of trypsinised cells. These results suggest that a significant portion of the conjugate with eight Arg residues could be bound at the outer surface of cell membrane more strongly than conjugate containing only four Arg residues. In addition, these data indicate clearly that the trypsin treatment is necessary to measure the correct amount of internalized conjugates.

In order to monitor the internalization, HL-60 sensitive and HL-60/MDR1 resistant cells were incubated with the solution of compounds including conjugates and DauSuc as control at different concentrations $(\mathrm{c}=0.16-100 \mu \mathrm{M})$ for $90 \mathrm{~min}$. The cells were treated with trypsin and fluorescence intensity as a function of concentration was measured. Results are depicted in Figure 5. 
a)

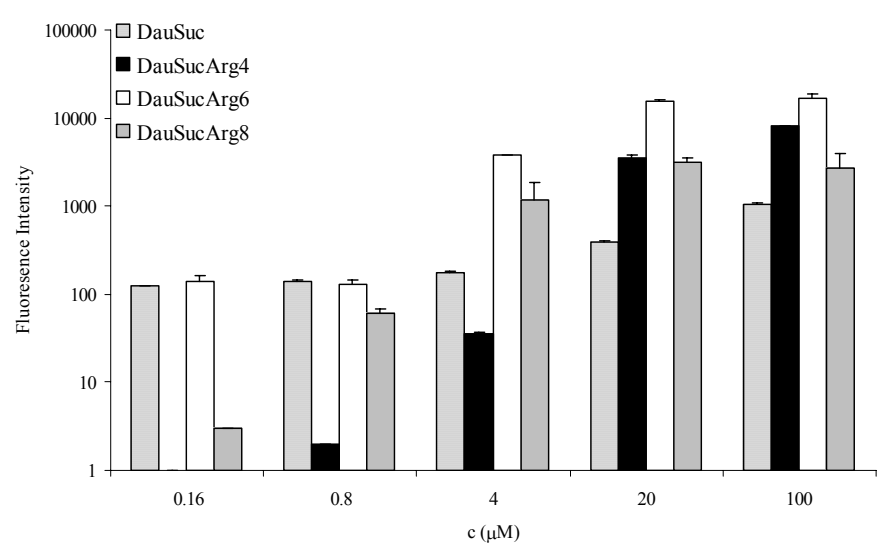

b)

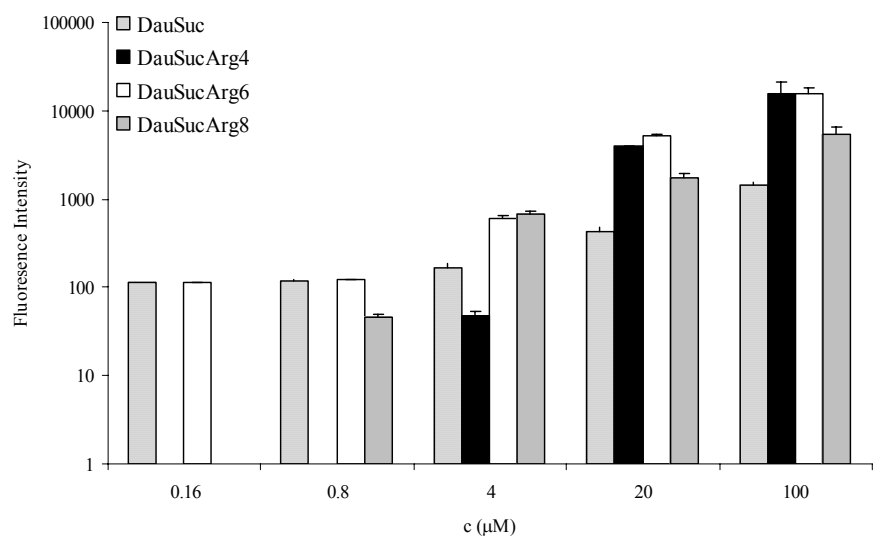

Figure 5. Uptake of octa-, hexa- and tetraarginine Dau-conjugate by sensitive (a) and MDR1 resistant (b) HL-60 cells.

We found that the internalization of all compounds by sensitive as well as resistant HL60/MDR1 cell, was concentration dependent (Figure 5). The internalization of Dau is very efficient and the saturable manner of the uptake (data not shown) might suggest the involvement of active transport process. The succinylation markedly decreased the cell-uptake. However, free DauSuc was taken up to some extent $\left(\mathrm{F}_{\text {mean }}=127-178\right)$ by sensitive HL-60 cells already at low concentrations $(\mathrm{c}=0.16-4 \mu \mathrm{M})$. From $\mathrm{c}=4 \mu \mathrm{M}$ the cellular uptake was increased without exhibiting a maximum level $\left(\mathrm{c}=20\right.$ and $100 \mu \mathrm{M}, \mathrm{F}_{\text {mean }}=385$ and 1056 , respectively). It may be that the positively charged amino group, under physiological condition, plays crucial rules in the internalization of Dau. However, further experiments are needed to support this hypothesis considering that structural modification of Dau could decrease its spectral properties ${ }^{31}$ (e.g. the $\varepsilon$ value). Therefore we have used DauSuc in our experiments as control.

The penetration of conjugates into sensitive cells was also dependent on concentration. The conjugation with oligoarginine increased the cell-uptake of DauSuc at $c=4 \mu \mathrm{M}$ in case of hexa- 
and octaarginine, whereas the tetraarginine conjugate was internalized only at high concentrations $\left(c=20 \mu \mathrm{M}\right.$ and $100 \mu \mathrm{M}, \mathrm{F}_{\text {mean }}=3507$ and 8038 , respectively), but with increasing efficiency. We found that the cell-uptake of hexa- and octaarginine conjugates was more efficient reached maximum around $\mathrm{c}=20 \mu \mathrm{M}$. Both conjugate internalized poorly at low concentration $(\mathrm{c}=0.16$ and $0.8 \mu \mathrm{M})$ and only from $\mathrm{c}=4 \mu \mathrm{M}$ showed significant cell-penetration. Comparing the translocation of the compounds the following order could be established at $\mathrm{c}=20$ $\mu \mathrm{M}:$ DauSucArg $_{6}>$ DauSucArg $_{4}=$ DauSucArg $_{8}>$ DauSuc. It is interesting to note that the tetraarginine conjugate penetrates more efficiency in sensitive HL/60 cells than the octaarginine variant at higher concentration $(c=100 \mu \mathrm{M})$. The hexaarginine conjugate showed the most effective penetration, but it was significantly better only at $\mathrm{c}=20 \mu \mathrm{M}$. Taken together, our results with oligoarginine conjugates show that at low concentration $(c=4 \mu \mathrm{M})$ the hexa- and octaarginine are the best transporter molecule, but at high concentrations (c $>20 \mu \mathrm{M})$ the tetraand hexaarginine become more effective.

In case of HL-60/MDR1 resistant cells the uptake was also concentration dependent, but no compounds exhibit platoing internalization. The pattern of cell-uptake of DauSuc essential is the same with that of found in sensitive cells. However, the fluorescence intensity vs concentration curves of conjugates were different in comparison with those of sensitive HL-60 cells. The tendency of the translocation of tetraarginine conjugate was very similar: marked internalization occurred at high concentrations ( $c=20 \mu \mathrm{M}$ and $100 \mu \mathrm{M}, \mathrm{F}_{\text {mean }}=3979$ and 15387, respectively). The ingestion of hexa- and octaarginine conjugates was very limited at lower concentrations $(\mathrm{c}=$ 0.16-4 $\mu \mathrm{M}$ ), but increased after incubation with cells at higher concentration. Based on data collected the following hierarchy were established at $\mathrm{c}=20 \mu \mathrm{M}$ (at almost the same at $\mathrm{c}=100$

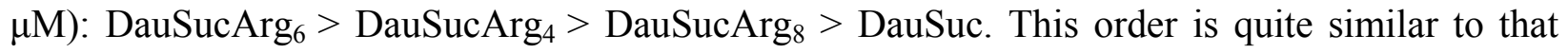
observed for sensitive cells, except that conjugate with four Arg residues was more efficiently translocated than DauSucArg.

In summary, in cellular uptake experiments our data indicate that both sensitive and MDR1 resistant HL-60 cells internalize oligoarginine conjugate of DauSuc. This process is highly dependent on concentration and also on the number of Arg residues present in the conjugate. All three conjugates penetrate much more efficiently by both cell lines than the free DauSuc compound at concentration above $4 \mu \mathrm{M}$. At low concentration we could not detect internalization of conjugate with tetraarginine, which is correlate well with data from measurement of celluptake of fluorescent tetraarginine. ${ }^{22}$

\section{Conclusion}

We described here the synthesis of a new set of compounds composed of Dau and of oligoarginine. We found that these conjugates are stable under certain conditions used for the in vitro cellular experiments. The conjugation of DauSuc with hexa- and octaarginine resulted in new compounds in which the in vitro antitumor activity on sensitive HL-60 cells was preserved. 
In resistant HL-60/MDR1 cells this effect was even increased as compared to DauSuc or maintained as compared to free Dau. The antiproliferative effect was dependent on the number of Arg residues: DauSucArg $8>$ DauSucArg $_{6}>>$ DauSucArg $_{4}$. Uptake studies show that conjugates are entering both sensitive and resistant HL-60 human leukemia cells. The uptake was only slightly dependent on cell types, but highly influenced by the concentration and by the number of Arg residues. Both cytotoxicity and cell-uptake data show that the hexaarginine and octaarginine transport Dau into cells efficiently at low concentration. In spite of the fact that the hexaarginine conjugate exhibited the highest internalization, its antitumor effect was lower than that of the octaarginine conjugate. It is one possibility that the internalization mechanism for hexa- and octaarginine conjugates is not the same therefore the uptake of DauSucArg 6 is higher than of DauSucArg 8 . It is also attractive to speculate that there are differences in the fate (metabolism, mechanism of action) of the two conjugates resulting in higher antitumor efficiency for the conjugate DauSucArg. Further studies to clarify the mechanism of action and also to investigate the in vivo antitumor effect of DauSucArg 8 and DauSucArg 6 are in progress.

\section{Experimental Section}

General Procedures. The crude products were purified on RP-HPLC using a semi-preparative Phenomenex Jupiter C18 column (250x10 mm I.D., $10 \mu \mathrm{m}$ silica, $300 \AA \AA$ pore size) (Torrance, CA, USA). Flow rate was $4 \mathrm{~mL} / \mathrm{min}$. Peaks were detected at $\lambda=220 \mathrm{~nm}$ at RT. A eluent was $0.1 \%$ TFA in water and B eluent was $0.1 \%$ TFA in acetonitrile-water (80:20, V/V).

Analytical RP-HPLC was performed on a Knauer (Herbert Knauer GmbH, Berlin, Germany) system using a Phenomenex SYNERGI MAX-RP column (250x4.6 mm I.D., $4 \mu \mathrm{m}$ silica, $80 \AA$ pore size) (Torrance, CA, USA) as a stationary phase. Linear gradient elution ( 0 min $0 \% \mathrm{~B} ; 5$ $\min 0 \% \mathrm{~B} ; 50 \min 90 \% \mathrm{~B}$ ) was generated. Flow rate of $1 \mathrm{~mL} / \mathrm{min}$ was applied at ambient temperature. Peaks were detected at $\lambda=220 \mathrm{~nm}$. The samples were dissolved in eluent $\mathrm{B}$.

Positive ion electrospray ionization mass spectrometric analysis was performed on a Bruker Esquire 3000 plus (Germany). The samples were dissolved in acetonitrile-water (50:50, V/V), containing $0.1 \%$ acetic acid.

${ }^{1} \mathrm{H}$ NMR spectra were recorded at $250 \mathrm{MHz}$ (250 MHz, Bruker) in DMSO-d6 solution with tetramethylsilane as an internal standard.

N-Succinyl-daunomycin (DauSuc). Daunomycin x HCl (20 mg, $35.5 \mu \mathrm{mol})$ was reacted with 2 eq. succinic anhydride $(7 \mathrm{mg}, 71.0 \mu \mathrm{mol})$ in presence of 3 eq. DIEA $(18 \mu \mathrm{L}, 106.5 \mu \mathrm{mol})$ as base in $1 \mathrm{~mL} \mathrm{DMF}$ at room temperature for $5 \mathrm{~h}$. The reaction mixture was diluted by $2 \mathrm{~mL}$ of eluent $\mathrm{A}$ and was purified by RP-HPLC. Gradient was: $0 \min 25 \% \mathrm{~B}, 5 \mathrm{~min} 25 \% \mathrm{~B}, 50 \mathrm{~min} 70 \% \mathrm{~B}$. Yield $81 \%$. Analytical RP-HPLC: $\mathrm{R}_{\mathrm{t}}=34.4 \mathrm{~min}$; ESI-MS: $628.1\left(\mathrm{M}+\mathrm{H}^{+}\right)^{+}, 650.2\left(\mathrm{M}+\mathrm{Na}^{+}\right)^{+}, 666.2$ $\left(\mathrm{M}+\mathrm{K}^{+}\right)^{+} .{ }^{1} \mathrm{H}-\mathrm{NMR} \delta_{\mathrm{H}}(250 \mathrm{MHz}, \mathrm{DMSO}-\mathrm{d} 6, \mathrm{TMS}): 7.88(1 \mathrm{H}, \mathrm{d}, \mathrm{H}-1), 7.88(1 \mathrm{H}, \mathrm{t}, \mathrm{H}-2), 7.62$ $(1 \mathrm{H}, \mathrm{t}, \mathrm{H}-3), 3.97$ (3H, s, 4-OMe), 4.91 (1H, t, H-7), 2.11 and $2.18\left(1+1 \mathrm{H}, \mathrm{dd}, \mathrm{H}_{2}-8\right), 5.53(1 \mathrm{H}$, 
s, 9-OH), 2.27 (3H, s, 9-COMe), 2.93 (2H, s, H2-10), 5.22 (1H, d, H-1'), 1.42 and 1.84 (1+1 H, dd and td, H-2'), 3.96 (1H, H-3'), 7.6 (1H, d, NH), 3.40 (1H, H-4'), 4.75 (1H, d, 4'-OH), 4.19 (1H, q, H-5'), 1.13 (3H, d, H3-6'), 2.31 (4H, t, $\left.\mathrm{CH}_{2}-\mathrm{CH}_{2}\right)$.

Oligoarginines. These compounds were synthesized manually by solid phase methodology on Rink-amide resin $(0.335 \mathrm{~g}, 0.73 \mathrm{mmol} / \mathrm{g})$. The amount of resin was used in three parts during the synthesis. The side chain protecting group of Arg was 2,2,4,6,7-pentamethyldihydrobenzofuran5 -sulfonyl group. Fmoc protection from the $N^{\alpha}$-amino groups was removed with $2 \%$ piperidine and 2\% 1,8-diazabicyclo[5.4.0] undec-7-ene (DBU) in DMF $(2+2+5+10 \mathrm{~min})$ followed by washing with DMF $(8 \times 0.5 \mathrm{~min})$ For coupling amino acid derivatives and $N, N^{\prime}-$ diisopropylcarbodiimide and HOBt dissolved in DMF were used in 3 molar excess for the resin capacity. The reaction proceeded for $60 \mathrm{~min}$ at $\mathrm{RT}$. Then, the resin was washed (DMF $2 \times 0.5$ $\mathrm{min})$, dichloromethane (DCM) $(3 \times 0.5 \mathrm{~min}))$. The efficiency of the coupling was checked by ninhydrin assay. ${ }^{33}$ After the removal of the last $N^{\alpha}$-Fmoc group, peptides was prepared by cleavage with $10 \mathrm{ml}$ TFA containing $0.75 \mathrm{~g}$ phenol, $0.5 \mathrm{ml}$ D.I. water, $0.5 \mathrm{ml}$ thioanisole and 0.25 ethandithiol as scavengers. Crude product was precipitated by dry diethyl-ether, dissolved in $10 \%$ acetic acid and freeze-dried. The crude peptide was purified by RP-HPLC as described above by using the following linear gradient: $0 \min 0 \% \mathrm{~B}, 5 \min 0 \% \mathrm{~B}, 50 \min 40 \% \mathrm{~B}$. The pure peptides were characterized by analytical RP-HPLC and MS (Table 1).

Daunomycin conjugates. The purified and characterized oligoarginines were reacted with DauSuc in DMF. $\operatorname{Arg}_{4}(5.3 \mathrm{mg}, 8.26 \mu \mathrm{mol})$, DauSuc (5.18 mg, $\left.8.26 \mu \mathrm{mol}\right)$, BOP (4.4 mg, 9.91 $\mu \mathrm{mol})$, HOBt $(1.5 \mathrm{mg}, 9.91 \mu \mathrm{mol})$ were dissolved in $0.5 \mathrm{ml} \mathrm{DMF}$ and DIEA $(1.4 \mu 1,8.26 \mu \mathrm{mol})$ was added to the solution. The reaction mixture was stirred overnight at RT. Then the reaction mixture was diluted by A eluent and purified on RP-HPLC using the following linear gradient: 0 $\min 10 \% \mathrm{~B}, 10 \mathrm{~min} 10 \% \mathrm{~B}, 55 \mathrm{~min} 70 \% \mathrm{~B}$. The reactions between $\mathrm{Arg}_{6}$ and DauSuc ( $\mathrm{Arg}_{6} 5 \mathrm{mg}$, $5.2 \mu \mathrm{mol}$; DauSuc $3.3 \mathrm{mg}, 5.2 \mu \mathrm{mol}$; BOP $2.3 \mathrm{mg}, 5.2 \mu \mathrm{mol}$; HOBt $0.6 \mathrm{mg}, 5.2 \mu \mathrm{mol}$; DIEA 1.9 $\mu \mathrm{l}, 5.2 \mu \mathrm{mol}$ in $0.5 \mathrm{~mL} \mathrm{DMF}$ ) and between $\mathrm{Arg}_{8}$ and DauSuc were performed similarly (Arg 10 mg, $7.9 \mu \mathrm{mol}$; DauSuc 5.5 mg, $7.9 \mu \mathrm{mol}$; BOP 3.5 mg, $7.9 \mu \mathrm{mol}$; HOBt $1.2 \mathrm{mg}$, $7.9 \mu \mathrm{mol}$; DIEA $2.9 \mu \mathrm{l}, 7.9 \mu \mathrm{mol}$ in $1 \mathrm{~mL} \mathrm{DMF})$. The purified conjugates were characterized by analytical RPHPLC and MS (Table 1). Yields: DauSucArg 4 31\%, DauSucArg 6 34\%, DauSucArg 8 29\%.

Stability studies. The stability of conjugates was studied by analytical RP-HPLC under conditions described above with detection at $\lambda=214,254$ and $490 \mathrm{~nm}$. The conjugates at $\mathrm{c}=1$ $\mathrm{mg} / \mathrm{mL}$ were dissolved in RPMI 1640 medium (Sigma, R-6504, $\mathrm{pH}=7.4$ ). Then the solutions were incubated at $37^{\circ} \mathrm{C}$. From the solution $30 \mu \mathrm{L}$ was injected after 0,90 and $180 \mathrm{~min}$.

Analysis of the in vitro antitumor effect. Sensitive as well as MDR1 resistant human leukemia (HL-60) cells were cultured in RPMI-1640 medium supplemented with 10\% fetal bovine serum. Cell culture was maintained at $37^{\circ} \mathrm{C}$ in a humidified atmosphere with $5 \% \mathrm{CO}_{2}$. To study the 
cytostatic effect of the conjugates $5 \times 10^{3}$ cells per well were plated on 96-well plates. After 24 hours incubation at $37^{\circ} \mathrm{C}$, cells were treated for 3 hours with the conjugates solved in serum-free RPMI-1640 medium. The compounds were used in the $2.6 \times 10^{-4}-10^{-2} \mu \mathrm{M}$ concentration range. Cells treated with serum-free medium for 3 hours were used for control. After incubation cells were washed twice with serum-free medium and cultured for 3 days in complete medium. On the 4th day 3-(4,5-dimethylthiazol-2-yl)-2,5-diphenyltetrazolium bromide (MTT)-assay was carried out. $45 \mu \mathrm{l} \mathrm{MTT-solution}(2 \mathrm{mg} / \mathrm{ml}$ ) was added to each well (final concentration: $367 \mu \mathrm{g} / \mathrm{ml}$ ). After 3.5 hours incubation purple crystals were formed by mitochondrial dehydrogenase enzyme of live cells. Cells were centrifuged for 5 minutes at $2000 \mathrm{rpm}$ and supernatant was removed. Crystals were dissolved in dimethyl sulfoxide (DMSO) and the optical density (OD) of the samples was measured at $\lambda=540$ and $\lambda=620 \mathrm{~nm}$ using ELISA Reader (Labsystems MS reader, Finland). $\mathrm{OD}_{620}$ was subtracted from $\mathrm{OD}_{540}$. The percent of cytostasis was calculated using the following equation:

$$
\text { Cytostasis } \%=\left[1-\left(\mathrm{OD}_{\text {treated }} / \mathrm{OD}_{\text {control }}\right)\right] \times 100,
$$

where $\mathrm{OD}_{\text {treated }}$ and $\mathrm{OD}_{\text {control }}$ correspond to the optical densities of treated and control cells, respectively. Cytostasis percent was illustrated in the function of concentration and $\mathrm{IC}_{50}$ values were determined ( $\mathrm{IC}_{50}$ is the concentration which inhibits $50 \%$ of the cells in the division.).

Analysis of cellular uptake. Sensitive as well as MDR1 resistant HL-60 human leukemia cells were cultured in RPMI-1640 medium supplemented with 10\% fetal bovine serum. Cell culture was maintained at $37^{\circ} \mathrm{C}$ in a humidified atmosphere with $5 \% \mathrm{CO}_{2}$. To study the cellular uptake of the conjugates and control compounds (Dau, DauSuc) $10^{5}$ cells per well were plated on 24well plates. After 24 hours incubation at $37^{\circ} \mathrm{C}$, cells were treated for 1.5 hours with the conjugates and control compounds solved in serum-free RPMI-1640 medium. The compounds were used in the $0.16-100 \mu \mathrm{M}$ concentration range. Cells incubated only with serum-free medium for $1.5 \mathrm{hrs}$ were used for control. After incubation cells were washed with HPMI and trypsinized for 10 minutes. (HPMI contains glucose, $\mathrm{NaHCO}_{3}, \mathrm{NaCl}, \mathrm{HEPES}, \mathrm{KCl}, \mathrm{MgCl}_{2}$, $\mathrm{CaCl}_{2}$ and $\mathrm{Na}_{2} \mathrm{HPO}_{4} \times 2 \mathrm{H}_{2} \mathrm{O}$ ). The effect of trypsin was terminated by HPMI supplemented with $10 \%$ fetal bovine serum and cells were moved from the plate to FACS-tubes. After washing, cells were re-suspended in HPMI. The increase of the fluorescence intensity of HL-60 cells after this preparation was monitored by flow cytometry (BD LSR II, BD Bioscience, San Jose, CA). Data were analyzed with FACSDiVa software.

For studying the effect of trypsin treatment the sensitive HL-60 cells were incubated with DauSucArg $_{4}$ or DauSucArg 8 conjugates at $\mathrm{c}=30 \mu \mathrm{M}$ as describe above.

\section{Acknowledgements}

This work was supported by Medichem 2 (1/A/005/2004), OTKA (K-68285) and ETT (43/2006). 


\section{References and Notes}

1. American Society Textbook of Clinical Oncology, 2nd Edn. Murphy G. P.; Lawrence, W. Jr.; Lenhard, R. E., Eds.; American Cancer Society: Atlanta, GA, 1995.

2. Wiernik, P. H.; Dutcher, J. P. Leukemia 1992, 6 (Suppl 1), 67.

3. Von Hoff, D. D.; Rozencweig, M.; Layard, M.; Slavik, M.; Muggia, F. M. Am. J. Med. 1977, 62, 200.

4. Riehm, H.; Biedler, J. L. Cancer Res. 1971, 31, 409.

5. Krishna, R.; Mayer, D. L. Eur. J. Pharm. Sci. 2000, 11, 265.

6. Teodori, E.; Dei, S.; Scapecchi, S.; Gualtieri, F. Il Farmaco 2002, 57, 385.

7. Hudecz, F.; Bánóczi, Z.; Csík, G. Med. Res. Rev. 2005, 25, 679.

8. Gaál, D.; Hudecz, F. Eur. J. Cancer. 1998, 34, 155.

9. Hudecz, F.; Reményi, J.; Szabó, R.; Kóczán, G.; Mező, G.; Kovács, P.; Gaál, D. J. Mol. Recognit. 2003, 16, 288.

10. Capobianco, M. L.; De Champdore, M.; Arcamone, F.; Garbesi, A.; Gulanvarch, D.; Arimondo, P. B. Bioorg. Med. Chem. 2005, 13, 3209.

11. Yamamoto, K.; Acton, E. M.; Henry, D. W. J. Med. Chem. 1972, 15, 872.

12. Masquelier, M.; Baurain, R.; Trouet, A. J. Med. Chem. 1980, 23, 1166.

13. Baurain, R.; Masquelier, M.; Deprez-De Campeneere, D.; Trouet, A. J. Med. Chem. 1980, 23, 1171.

14. Langer, M.; Kratz, F.; Rothen-Rutishauser, B.; Wunderli-Allenspach, H.; Beck-Sickinger, G. A. J. Med. Chem. 2001, 44, 1341.

15. Roby, P.; Page, M. Oncology Reports 1996, 3, 175.

16. Ingallinella, P.; Di Marco, A.; Taliani, M.; Fattori, D.; Pessi, A. Bioorg. Med. Chem. Lett. 2001, 11, 1343.

17. Krauss, U.; Kratz, F.; Beck-Sickinger, G. A. J. Mol. Recognit. 2003, 16, 280.

18. Daussin, F.; Boschetti, E.; Delmotte, F.; Monsigny, M. Eur. J. Biochem. 1988, 176, 625.

19. Shen, W. C.; Ryser, H. J. P. Biochem. Biophys. Res. Commun. 1981, 102, 1048.

20. Hudecz, F.; Clegg, A. J.; Kajtár, J.; Embleton, J. M.; Szekerke, M.; Baldwin, W. R. Bioconjugate Chem. 1992, 3, 49.

21. Arnold, R. J., Jr. Methods Enzymol. 1985, 112, 270.

22. Rodrigues, C. A. P.; Roth, T.; Fiebig, H. H.; Unger, C.; Mülhaupt, R.; Kratz, F. Bioorganic \& Medicinal Chem. 2006, 14, 4110.

23. Patel, L.; N; Zaro, J. L.; Shen, W. C. Pharm. Res. 2007 Apr 19, online.

24. Mitchell, D. J.; Kim, D. T.; Steinman, L.; Fathman, C. G.; Rothbard, J. B. J Pept. Res. 2000, 56,318 .

25. Futaki, S.; Nakase, I.; Tadokoro, A.; Takeuchi, T.; Jones, A. T. Biochem. Soc. Trans. 2007, 35, 784.

26. Futaki, S.; Suzuki, T.; Ohashi, W.; Yagami, T.; Tanaka, S.; Ueda, K.; Sugiura, Y. J. Biol. Chem. 2001, 276, 5836. 
27. Rothbard, J. B.; Garlington, S.; Lin, Q.; Kirschberg, T.; Kreider, E.; McGrane, P. L.; Wender, P. A.; Khavari, P. A. Nat. Med. 2000, 6, 1253.

28. Miklán, Zs.; Szabó, R.; Zsoldos-Mády, V.; Reményi, J.; Bánóczi, Z.; Hudecz, F. Biopolymers. 2007, 88, 108.

29. Castro, B.; Dormoy, J. R,; Eving, G.; Selve, C. Tetrahedron Lett. 1975, 14, 1219.

30. Hudecz, F.; Reményi, J.; Hegedüs, T.; Kovács, P.; Szabó, B.; Sarkadi, B.; Gaál, D. Peptides: Peptide Revolution: Genomics, Proteomics and Therapeutics. Proceedings of the 18th American Peptide Symposium; Chorev, M.; Sawyer, T. K. Eds.; American Peptide Society, 2004; pp 815.

31. Reményi, J.; Csík, G.; Kovács, P.; Reig, F.; Hudecz, F. Biochim. Biophys. Acta 2006, 1758, 280.

32. Richard, J. P.; Melikov, K.; Vives, E.; Ramos, C.; Verbeure, B.; Gait, M. J.; Chernomordik, L. V.; Lebleu, B. J. Biol. Chem. 2003, $278,585$.

33. Kaiser, E.; Colescott, R. L.; Bossinger, C. D.; Cook, P. I. Anal. Biochem. 1970, 34, 595. 